\title{
Dosimetry Study of VAMT Planning Based onMonaco Treatment Planning System and Tomo Planning System Based on Helical Tomography in Volume-Modulated Intensity-Modulated Radiation Therapy for Cervical Cancer
}

\author{
Mengyuan $\mathrm{Si}^{1} \mathcal{Q}$,Yuqin Wen ${ }^{1} \mathcal{Q}$, Ruiqiang Song ${ }^{1}$
}

${ }^{1}$ College of Instrument \&Meter Engineering,Chengdu University of Technology, Chengdu 610059,China

\begin{abstract}
To compare and analyze the effects of VAMT plan based on Monaco treatment plan system and Tomo treatment plan system based on spiral tomography system on cervical cancer dosimetry. [Methods] Ten patients with cervical cancer who received cervical cancer radiotherapy from September 2017 to May 2018 were selected, using the VAMT plan based on MonacoTPS and the Tomo plan based on TomoHTMV2.0.5 TPS. Target dosimetry index, endangered organ dosimetry index, machine jumps, and patient treatment time. [Result] The conformity index CI of the target area of the TOMO plan is $(0.85 \pm 0.02)$ and the uniformity index $\mathrm{HI}$ is $(0.07 \pm 0.01)$, which are better than the CI $(0.80 \pm 0.01)$ and $\mathrm{HI}(0.10 \pm 0.01)$ of the VAMTMonaco plan. The difference is highly statistically significant (p $<0.001)$. The TOMO plan has significantly reduced the V30 and V40 of OAR, especially for the bladder. The MU $(6698 \pm 473)$ and DT $(473 \pm 33)$ required for the TOMO plan were significantly larger than the MU $(1655 \pm 101)$ and DT $(167 \pm 16)$ for the VAMTMonaco plan, and the differences were highly statistically different $(\mathrm{p}<0.001)$. [Conclusion] Compared with the VAMTMonaco plan, the TOMO plan has better conformity and uniformity of the target area, and the normal tissues around the target area receive lower doses, which is better for protecting organs at risk, especially for bladder protection. However, in OARV20 and V10, VAMTMonaco plan is better than TOMO plan, and MU and DT are smaller than TOMO plan, and the treatment rate is higher than TOMO plan. From a dosimetric point of view, using the TOMO plan under certain conditions is a better choice.
\end{abstract}

Keywords: Cervical Cancer, Spiral Tomography,Volume Rotation Intensity Modulation,Dosimetry

\section{Introduction}

Cervical cancer is one of the most common cancers among women in developing countries, and its incidence has gradually increased in recent years. At the same time, the incidence has gradually decreased. It is estimated that China has 98900 new cases and 30.5 million deaths each year [1-2]. At present, a comprehensive treatment plan mainly based on surgery and radiotherapy, supplemented by chemotherapy is used for treatment [3]. With the development of technology, Helical Tomotherapy (TOMO) technology and Volume Modulated Arc Therapy (VMAT) technology have increasingly become the preferred technologies for cervical cancer treatment. Compared with traditional fixed-field IMRT technology, TOMO and VAMT technologies have a higher degree of freedom in the direction of the radiation field, which is more conducive to protecting the radiation dose of normal tissues, and can also target tumors. Zone produces higher dose conformation [4]. TOMO and VAMT are rotary treatments, and are not restricted by angles during treatment. However, the treatment methods of the two are different. The TOMO technology is the integration of spiral CT and linear accelerator, which is combined with linear accelerator, and is integrated intensity-modulated radiotherapy (IMRT), image guidance, and dose. Guidance and other technologies in one, it has obvious advantages in the treatment of tumor target area larger, longer, and complex shape. The equipment of VAMT technology is the same as that of traditional accelerators. Compared with fixed fluids, VAMT technology has a shorter treatment time, fewer machine hops, and has obvious advantages in Linchuan [5]. Due to the differences in VAMT produced by different commercial radiation planning system (TPS), this article compares and analyzes the dosimetric differences between the TOMO plan of 10 patients with cervical cancer and the VAMT plan made by Monaco TSP and the machine bar. Count and treatment time.

This article is published under the terms of the Creative Commons Attribution License 4.0 Author(s) retain the copyright of this article. Publication rights with Alkhaer Publications. Published at: http://www.ijsciences.com/pub/issue/2020-05/

DOI: 10.18483/ijSci.2320; Online ISSN: 2305-3925; Print ISSN: 2410-4477 
Dosimetry Study of VAMT Planning Based onMonaco Treatment Planning System and Tomo Planning System Based on Helical Tomography in Volume-Modulated Intensity-Modulated Radiation Therapy for Cervical Cancer

1.Materials and Methods

2.1Case selection and general information

Ten patients with advanced stage III and IV cervical cancer radiotherapy were selected. The pathological types were all squamous cell carcinomas, aged 36 to 47 years, with a median age of 41 years. According to the 2009 International Obstetrics and Gynecology Association FIGO staging criteria: 3 cases were stage IA, 4 cases were stage IB, and 3 cases were stage IIB.

\subsection{Target area sketch}

The same experienced physician outlines the cervical gross tumor area (GTV) and clinical target volume (CTV) according to the guidelines of the National Radiotherapy Oncology Group (RTOG) for cervical cancer postoperative radiotherapy clinical target volume delineation guidelines, and The CTV is placed 6 to $8 \mathrm{~mm}$ from the front, back, left and right, and 8 to $10 \mathrm{~mm}$ from the up and down direction, respectively, as the planning target volume (PTV). OAR includes: small intestine, rectum, bladder, and pelvic bone marrow (PBM). PBM includes the sacrum, sacrum, hip bone, ischium, pubic bone, upper femur, and bilateral femoral head.

\subsection{Target prescription dose and organ endangered limit}

The target area prescription dose was $45 \mathrm{~Gy}$, and the segmentation method was $1.8 \mathrm{~Gy} /$ time, 5 times / week, and 1 time / day. Prescription dose requirements: The minimum $95 \%$ PTV dose reaches $45 \mathrm{~Gy}$, there are no cold spots in the CTV, no hot spots outside the PTV, and no hot spots in the rectum and posterior bladder wall. Tolerated doses for organ-endangered patients require $\mathrm{PBMV} 30<50 \%$, small intestine $\mathrm{V} 30<50 \%$, rectal V40 $<50 \%$, and bladder V45 $<50 \%$.

\subsection{Radiotherapy plan design}

The same physicist used Monaco TSP and TomoHTMV2.0.5 TPS to make the VAMT plan and the TOMO plan, of which the VAMT plan uses double arc coplanar rotation irradiation $\left(181^{\circ} \sim 179^{\circ}\right.$ and $179^{\circ} \sim$ $181^{\circ}$ ), and the ray energy is $6 \mathrm{MeV}$. TOMO plans to use $360^{\circ}$ rotation irradiation, and the optimization parameters are the initial optimization parameters.

\subsection{Plan evaluation}

Compare the cross-section dose distribution with the target area and the dose-volume histogram (DVH) of the OAR, and evaluate the target area and the OAR. Target area measurement and evaluation indicators include: $5 \%, 50 \%, 90 \%, 95 \%, 98 \%$ of the volume of the target area D5, D50, D90, D95, D98, target dose conformity index (CI), Uniformity Index (HI), 5\%, $30 \%, 50 \%, 70 \%, 90 \%, 100 \%$ of the prescribed dose in vivo volume to target volume ratio NTV5 / PTV, NTV30 / PTV, NTV50 / PTV, NTV70 / PTV, NTV90 / PTV, NTV100 / PTV.

$C I=\frac{V_{T, r e f}}{V_{T}} \times \frac{V_{T, r e f}}{V_{r e f}}$

In the formula:

VT, ref is the volume at which the target area receives a dose equal to or greater than the prescribed dose

Vref is the volume at which the received dose is equal to or greater than the prescribed dose

VT target volume

The ideal value for $\mathrm{CI}$ is 1 .

$H I=\frac{D_{2 \%}-D_{98 \%}}{D_{50 \%}}$

among them:

$\mathrm{D} 2 \%$ is the dose received at the $2 \%$ target area

D98\% is the dose received at $98 \%$ of the target area $\mathrm{D} 50 \%$ is the dose received at $50 \%$ of the target area The ideal value of $\mathrm{HI}$ is 0

(2) OAR dose evaluation indicators include the dose volumes V10, V20, V30, and V40, and Dmax and Dmean of PBM, small intestine, rectum, and bladder;

(3) Evaluate accelerator machine jumps (MU) and patient treatment time (DT).

\subsection{Statistical methods}

SPSS 26.0 statistical analysis software was used for data entry, collation and statistical analysis. Measurement data are expressed as mean \pm standard deviation $(x \pm s)$. Differences in target area dosimetric indexes, in vivo exposure dose distribution differences, and OAR irradiation dosimetry index differences between the two plans are used after normal distribution tests Paired t-test analysis was performed, and $\mathrm{P}<0.05$ showed statistically significant differences; $\mathrm{P}<0.001$ was considered highly statistically significant.

\section{Result}

3.1Target dose comparison

Table 1 Comparison of target dose parameters $\quad(\bar{x} \pm s)$

\begin{tabular}{|c|c|c|c|c|c|}
\hline Group & VAMT $_{\text {monaco }}$ & TOMO & Relative deviation & t value & p value \\
\hline CI & $0.80 \pm 0.01$ & $0.85 \pm 0.02$ & $6.25 \%$ & -6.186 & $<0.001$ \\
\hline HI & $0.10 \pm 0.01$ & $0.07 \pm 0.01$ & $-30.00 \%$ & 6.012 & $<0.001$ \\
\hline D98(Gy) & $44.14 \pm 0.38$ & $43.57 \pm 2.42$ & $-1.29 \%$ & 0.766 & 0.463 \\
\hline D95(Gy) & $45.20 \pm 0.36$ & $44.67 \pm 1.54$ & $-1.17 \%$ & 1.116 & 0.293 \\
\hline D90(Gy) & $45.70 \pm 0.23$ & $45.80 \pm 0.36$ & $0.21 \%$ & -0.745 & 0.475 \\
\hline D50(Gy) & $44.26 \pm 9.20$ & $46.66 \pm 0.75$ & $5.42 \%$ & -0.837 & 0.424 \\
\hline D5(Gy) & $49.02 \pm 0.60$ & $48.00 \pm 1.23$ & $-2.08 \%$ & -2.364 & 0.042 \\
\hline
\end{tabular}


Dosimetry Study of VAMT Planning Based onMonaco Treatment Planning System and Tomo Planning System Based on Helical Tomography in Volume-Modulated Intensity-Modulated Radiation Therapy for Cervical Cancer

This study shows that both the Tomo radiotherapy plan and the VAMTmonaco radiotherapy plan can meet the clinical needs for target area coverage. The TOMO plan shown in Table 1 has a lower D98 and D5 than the VAMTMonaco plan, and D98 is not statistically significant. (P> 0.05), D5 was statistically significant $(\mathrm{p}<0.05)$; the target areas of the two plans were close to D95, and the relative deviation between the two was only $-1.17 \%$, no statistical difference ( $p>0.05$ ); the TOMO plan was at Compared with VAMTMonaco plan, D90 and D50 were all improved, there was no statistical difference ( $p>0.05)$; the conformity CI and uniformity $\mathrm{HI}$ of TOMO plan were better than VAMTMonaco plan, and they were statistically significant $(\mathrm{p}<0.05)$.

\subsection{Comparison of dose distributions in the body}

Table 2 Comparison of dose distribution in the body $(\bar{x} \pm s)$

\begin{tabular}{|c|c|c|c|c|c|}
\hline Group & VAMT $_{\text {Monaco }}$ & TOMO & $\begin{array}{c}\text { Relative } \\
\text { deviation }\end{array}$ & t value & $\mathrm{p}$ value \\
\hline NTV100/PTV & $1.14 \pm 0.02$ & $1.07 \pm 0.03$ & $-6.14 \%$ & 8.72 & $<0.001$ \\
\hline NTV90/PTV & $1.58 \pm 0.04$ & $1.55 \pm 0.05$ & $-1.90 \%$ & 2.03 & 0.073 \\
\hline NTV70/PTV & $2.53 \pm 0.07$ & $2.46 \pm 0.11$ & $-2.77 \%$ & 2.52 & 0.033 \\
\hline NTV50/PTV & $4.67 \pm 0.28$ & $4.31 \pm 0.26$ & $-7.71 \%$ & 4.478 & 0.002 \\
\hline NTV30/PTV & $9.11 \pm 1.17$ & $9.38 \pm 0.99$ & $2.96 \%$ & -1.943 & 0.084 \\
\hline NTV5/PTV & $14.65 \pm 2.05$ & $16.21 \pm 2.45$ & $10.65 \%$ & -9.331 & $<0.001$ \\
\hline
\end{tabular}

As shown in Table 2, the NTV100 / PTV of the TOMO plan and the VAMTMonaco plan are $(1.07 \pm 0.03)$ and $(1.14 \pm 0.02)$, respectively. The NTV100 / PTV of the TOMO plan is closer to 1 , which indicates that the TOMO plan receives prescriptions throughout the body. The area exposed to the dose was significantly smaller than the VAMTMonaco plan, and the difference was highly statistically significant $(\mathrm{p}<0.001)$. Both plans received $>50 \%$ of the prescribed dose volume in vivo, and the TOMO plan was significantly smaller than the
VAMTMonaco plan. NTV100 / PTV, NTV70 / PTV, and NTV50 / PTV were statistically significant (p $<0.05$ ), and NTV90 / PTV was not statistically significant Significance ( $>>0.05$ ). Compared with the VAMTMonaco plan, the TOMO plan has increased in NTV30 / PT and NTV5 / PTV, with statistical differences $(p<0.05)$. The TOMO plan has a smaller dose coverage volume in the body $>22.5 \mathrm{~Gy}$ and a larger dose coverage volume in the body $<22.5 \mathrm{~Gy}$.

3.3Comparison of Dangerous Organ Dose Distributions

Table 3 Comparison of exposure parameters of endangered organs $(\bar{x} \pm s)$

\begin{tabular}{|c|c|c|c|c|c|c|c|c|}
\hline \multicolumn{3}{|c|}{ Group } & \multirow{2}{*}{$\begin{array}{l}\text { VAMTMo } \\
\text { naco } \\
4\end{array}$} & \multirow{2}{*}{\multicolumn{2}{|c|}{$\begin{array}{l}\text { TOMO } \\
90.37 \pm 4.3 \\
\end{array}$}} & \multirow{2}{*}{$\begin{array}{c}\begin{array}{c}\text { Relative } \\
\text { deviation }\end{array} \\
-3.12 \\
\end{array}$} & \multirow{2}{*}{$\begin{array}{l}\text { t value } \\
-1.91\end{array}$} & \multirow{2}{*}{$\begin{array}{l}\mathrm{p} \text { value } \\
0.09\end{array}$} \\
\hline \multirow{6}{*}{ PBM } & & $\mathrm{V} 10(\%)$ & & & & & & \\
\hline & & $\mathrm{V} 20(\%)$ & $66.86 \pm 3.1$ & $\varepsilon$ & $68.13 \pm 4.2$ & 3.40 & 0.89 & 0.40 \\
\hline & & V30(\%) & $39.72 \pm 4.4$ & 7 & $37.92 \pm 2.6$ & -4.50 & -1.91 & 0.09 \\
\hline & & $\mathrm{V} 40(\%)$ & $19.16 \pm 2.7$ & 5 & $17.18 \pm 1.5$ & -10.33 & -3.89 & $<0.001$ \\
\hline & ) & $\operatorname{Dmax}(\mathrm{Gy}$ & $50.68 \pm 0.8$ & & $47.88 \pm 0.2$ & -5.52 & -9.40 & $<0.001$ \\
\hline & $\mathrm{y}$ & Dmean $(\mathrm{G}$ & $26.87 \pm 1.1$ & & $26.45 \pm 0.9$ & -1.56 & -.1 .40 & 0.20 \\
\hline \multirow{6}{*}{ 小肠 } & & V10(\%) & $84.91 \pm 8.5$ & & $97.11 \pm 4.3$ & 14.37 & 5.82 & $<0.001$ \\
\hline & & V20(\%) & $48.30 \pm 9.4$ & & $50.52 \pm 8.3$ & 4.60 & 0.84 & 0.42 \\
\hline & & V30(\%) & $21.29 \pm 6.5$ & & $19.22 \pm 6.7$ & -9.70 & -2.84 & 0.02 \\
\hline & & V40(\%) & $7.50 \pm 4.04$ & & $6.61 \pm 4.14$ & -11.87 & -1.69 & 0.13 \\
\hline & ) & $\operatorname{Dmax}(\mathrm{Gy}$ & $50.27 \pm 1.2$ & & $47.53 \pm 0.7$ & -5.34 & -10.30 & $<0.001$ \\
\hline & $\mathrm{y}$ & Dmean $(\mathrm{G}$ & $21.21 \pm 2.4$ & & $21.11 \pm 3.3$ & -0.47 & -0.11 & 0.91 \\
\hline \multirow{5}{*}{ 直肠 } & & V10(\%) & $96.64 \pm 5.2$ & & $98.84 \pm 2.1$ & 2.28 & 2.00 & 0.08 \\
\hline & & $\mathrm{V} 20(\%)$ & $84.64 \pm 8.6$ & & $85.12 \pm 10$ & 0.57 & 0.15 & 0.88 \\
\hline & & V30(\%) & $61.97 \pm 10$ & & $60.19 \pm 13$ & -9.70 & -0.60 & 0.57 \\
\hline & & $\mathrm{V} 40(\%)$ & $41.39 \pm 10$ & & $37.37 \pm 12$ & -9.71 & -1.49 & 0.17 \\
\hline & ) & $\operatorname{Dmax}(\mathrm{Gy}$ & $51.07 \pm 0.8$ & 6 & $47.54 \pm 0.4$ & -6.91 & -10.72 & $<0.001$ \\
\hline
\end{tabular}


Dosimetry Study of VAMT Planning Based onMonaco Treatment Planning System and Tomo Planning System Based on Helical Tomography in Volume-Modulated Intensity-Modulated Radiation Therapy for Cervical Cancer

\begin{tabular}{|c|c|c|c|c|c|c|c|c|}
\hline & y) & Dmean $(\mathrm{G}$ & & $33.89 \pm 2.9$ & $\begin{array}{ll} & 33.41 \pm 3.3 \\
\end{array}$ & -1.42 & -0.66 & 0.53 \\
\hline \multirow{6}{*}{ 膀胱 } & & $\mathrm{V} 10(\%)$ & 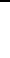 & $93.32 \pm 5.0$ & $\begin{array}{ll} & 100.00 \pm 0 .\end{array}$ & 7.16 & 4.22 & $<0.001$ \\
\hline & & V20(\%) & 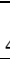 & $65.10 \pm 9.4$ & $\begin{array}{ll} & 64.81 \pm 7.2 \\
\end{array}$ & -0.45 & -0.11 & 0.92 \\
\hline & & $\mathrm{V} 30(\%)$ & , & $44.87 \pm 8.7$ & $9 \quad 34.65 \pm 6.2$ & -22.78 & -7.97 & $<0.001$ \\
\hline & & $\mathrm{V} 40(\%)$ & 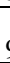 & $27.80 \pm 7.8$ & $\begin{array}{ll} & 19.47 \pm 5.8 \\
1 & \end{array}$ & -29.96 & -7.60 & $<0.001$ \\
\hline & ) & $\operatorname{Dmax}(\mathrm{Gy}$ & 3 & $51.14 \pm 0.8$ & $\begin{array}{ll} & 47.86 \pm 0.3 \\
\end{array}$ & -6.41 & -12.84 & $<0.001$ \\
\hline & y) & Dmean $(\mathrm{G}$ & 5 & $28.38 \pm 3.0$ & $8 \quad 27.16 \pm 1.5$ & -4.30 & -2.08 & 0.07 \\
\hline
\end{tabular}

Table 3 shows that the TOMO plan and the VAMTMonaco plan have better protection for OAR than the TOMO plan. In terms of dose distribution of PBM, small intestine, rectum, and bladder, the TOMO plan has significantly decreased compared to the VAMTMonaco plan in V30, V40, Dmax, and Dmean. Among them, the PBMV40 and Dmax have decreased by $10.33 \%$ and $5.52 \%$, respectively, and the small intestine Dmax has decreased. 5.34\%, rectal Dmax decreased by $6.91 \%$, and bladder V30, V40, and Dmax decreased by $22.78 \%, 29.96 \%$, and $6.41 \%$, respectively, with a high statistical significance $(\mathrm{p}<0.001)$. TOMO plan has improved in PBMV20, small intestine V10, V20, rectal V10, V20, and bladder V10 compared to VAMTMonaco plan. Among them, the small intestine V10 and bladder V10 increased by $14.37 \%$ and $7.16 \%$, respectively, and were highly statistically significant ( $\mathrm{p}$ $<0.001)$.

\subsection{Comparison of Mu and DT}

Table 4 Comparison of machine jumps and treatment time $(\bar{x} \pm s)$

\begin{tabular}{|c|c|c|c|c|}
\hline Group & VAMT $_{\text {Monaco }}$ & TOMO & t value & p value \\
\hline MU & $1655 \pm 101$ & $6698 \pm 473$ & 30.87 & $<0.001$ \\
\hline DT & $167 \pm 16$ & $473 \pm 33$ & 31.54 & $<0.001$ \\
\hline
\end{tabular}

Table 4 shows that the average number of machine hops treated by the TOMO plan $(6698 \pm 473)$ and the average number of machine hops of the VAMTMonaco plan $(1655 \pm 101)$ increased significantly, with a high statistical significance $(\mathrm{p}<0.001)$. The average treatment time of the TOMO plan $(473 \pm 33)$ was also significantly longer than the average treatment time of the VAMTMonaco plan $(167 \pm 16)$, and the difference was highly statistically significant $(\mathrm{p}<0.001)$.

\section{Discuss}

Studies have shown that both TOMO and VAMT technologies enable PTV to achieve prescription dose coverage [6-7]. The differences between TOMO technology and VAMT technology are mainly manifested in the conformity and uniformity of the target area, the speed of dose fall outside the target area, the danger to organ protection, the number of machine jumps and the treatment time. For cervical cancer, the large volume of the irregular pelvis, the close or coincidence of PTV and OAR boundaries, and the large change in the shape of the target area along the longitudinal axis of the human body all affect the treatment effect of the patient. The prescription dose coverage of D95 for the two treatment plans is similar. The TOMO plan is smaller than the VAMTMonaco plan at D5 and D98; the target area conformity and uniformity of the TOMO plan are better than that of the VAMTMonaco plan, and the fitness index parameters are increased by $6.25 \%$ ( $\mathrm{P}<0.001)$, the uniformity index parameter was reduced by $30.00 \%(\mathrm{p}<0.001)$; in the comparison of $>50 \%$ of the prescribed dose volume received in the body, the TOMO plan was reduced by $7.71 \%$ compared to the VAMTMonaco plan ; Shows that TOMO can reduce high doses in the target area, while reducing hot spots. The TOMO plan can make the dose drop outside the target area more steep, and improve the conformity and uniformity of the target area.

In the protection of endangered organs, the TOMO plan effectively controlled the maximum and average doses of each endangered organ, reducing V40 and V30 of each endangered organ. In particular, the radiation dose of the bladder was reduced, and the radiation damage of patients with acute cystitis was reduced [8]. With the exception of PBMV10 and bladder V20, the TOMO plan in V20 and V10 at various organ endangered levels has been improved compared to the VAMTMonaco plan. TomoTherapy is a system designed as image-guided intensity-modulated radiotherapy, which will generate an additional dose of approximately $0.6 \sim 2 \mathrm{cGy}$ for verification of patient positioning and tumor size, shape and location [9]. Although these additional doses will increase the low-dose absorption of endangered organs, they can enable patients to obtain images in real time during treatment, ensuring that the patient's position is consistent with the planned design during treatment, thereby improving treatment accuracy and endangering organ protection. In addition to the extra dose generated by image guidance, which can endanger low-dose absorption of organs, the TOMO plan performs better in conformity than the VAMTMonaco 
Dosimetry Study of VAMT Planning Based onMonaco Treatment Planning System and Tomo Planning System Based on Helical Tomography in Volume-Modulated Intensity-Modulated Radiation Therapy for Cervical Cancer

plan. The cost of improving the conformity is to increase the low-dose absorption of normal tissues [10-12 ].

The TOMO plan has a longer treatment time. Effective treatment time not only affects the comfort of patients during treatment, but also affects the treatment efficiency. The Mu of the TOMO plan is significantly increased compared to the VAMTMonaco plan. The reason is that Tomo Therapy has tens of thousands of subfields distributed in a $360^{\circ}$ spiral irradiation. It is not limited by a specific angle during treatment. More subfield angles mean that When designing a treatment plan, there is more modulation capability, and the treatment accuracy is higher. The better the target area dose conformity, the lower the risk of complications in normal tissues.

In summary, TOMO technology has better CI and HI than VAMT technology; it has better protection for endangered organs, but because the TOMO plan is significantly larger than the VAMT plan in terms of MU and DT, the treatment rate and the use of radiation The rate is not high. From a dosimetric point of view, using the TOMO plan is a better choice when the summation allows.

\section{References}

1. radiation therapy in definitive radiotherapy for 1433 patients with cervical cancer

2. Chen Chunlin. Clinical diagnosis and big data of cervica cancer in China $[\mathrm{J}]$. Chinese Journal of Practical Gynecology and Obstetrics.

3. Jie Junqin, Xiao Liping, Chen Danni, et al. Construction and verification of Markov model for natural process of cervical cancer in Chinese urban women\% Markov model construction and verification of natural process of cervical cancer in Chinese urban women [J]. China Health Resources, 2019 (3).

4. Cheng Tingting, Zhang Yanqiu, Zhang Zijian, et al. Dosimetric comparison of spiral tomography and volume rotation IMRT for advanced nasopharyngeal carcinoma $[\mathrm{J}]$. Chinese Journal of Medical Physics, 2018 (9): 1005-1011.

5. Zhang Yanqiu, Han Ameng, Li Jinwang, et al. Dosimetry study of CRT + ARC technology in radiotherapy for patients with "T" shaped target area of lung cancer [J] Chinese Journal of Medical Physics, 2017, 34 (7): 712-718.

6. Pan Linfei, Wang Binbing, Shan Guoping, et al. Study on dosimetry of helical tomotherapy and volumetric modulated arc therapy in cervical cancer $[\mathrm{J}]$. China Medical Herald, 2017, 014 (011): 95-98, Seal 4.

7. Dosimetric Study of Volume Rotational Intensity Modulation and Spiral Tomography for Locally Advanced Laryngeal Cancer and Hypharyngeal Cancer [J]. Journal of Practical Cancer (Issue 1): 120-123.

8. Michalski J M , Gay H , Jackson A et al. Radiation DoseVolume Effects in Radiation-Induced Rectal Injury[J] international journal of radiation oncology biology physics, 2010, 76(3-supp-S):0-0.

9. Sterzing F, Schubert K, Srokaperez G et al. Helical tomotherapy. Experiences of the first 150 patients in Heidelberg[J]. strahlentherapie und onkologie, 2008, 184(1):8-14.

10. Small W, Mell L K , Anderson P, et al. Consensus Guidelines for Delineation of Clinical Target Volume for Intensity-Modulated Pelvic Radiotherapy in Postoperative Treatment of Endometrial and Cervical Cancer[J]. International Journal of Radiation Oncology*Biology*Physics, 2008, 7

11. Sterzing F, Schubert K, Sroka-Perez G , et al. Helical Tomotherapy $[\mathrm{J}]$. Strahlentherapie und Onkologie, 2008 , 184(1):8-14.

12. Sterzing F, Schubert K, Srokaperez G , et al. Helical tomotherapy. Experiences of the first 150 patients in Heidelberg $[\mathrm{J}]$. strahlentherapie und onkologie, 2008 , 184(1):8-14

13. Cem Onal, Yemliha Dölek, Berna Akkuş Yıldırım Dosimetric comparison of 3-dimensional conformal radiotherapy, volumetric modulated arc therapy, and helical tomotherapy for postoperative gastric cancer patients[J]. Japanese Journal of Radiology, 2018, 36(1):30-39 